\title{
Synthese, Struktur, Tautomerie und Reaktivität von Methantrisamidinen
}

\author{
Benjamin Gutschank, Stephan Schulz, * Michael Marcinkowski, Georg Jansen, Heinz Bandmann, \\ Dieter Bläser, Christoph Wölper
}

$\mathrm{N}, \mathrm{N}$-chelatisierende, monoanionische Amidinatliganden wurden im letzten Jahrzehnt intensiv untersucht, da ihre sterischen und elektronischen Eigenschaften sehr einfach variiert werden können, ${ }^{[1]}$ was sie für die Synthese maßgeschneiderter Komplexe in der Katalyse und den Materialwissenschaften begünstigt. ${ }^{[2]} \mathrm{Zu}$ unserer Überraschung sind multifunktionale Liganden mit mehr als zwei Amidinatliganden, sogenannte Polyamidine, dagegen weitgehend unbekannt, obwohl diese vielversprechende Kandidaten für die Synthese (hetero)multimetallischer Komplexe mit verbesserten katalytischen Eigenschaften sind. Zudem wurden neutrale aromatische Tetraamidine in der Krebsforschung aufgrund ihrer Antiproteinaseaktivität untersucht. ${ }^{[3]}$ Leider wurden bis heute nur einige wenige, nahezu ausnahmslos phenylverbrückte, Polyamidine synthetisiert, während die Zahl der Polyamidine, in denen die Amidingruppen über ein einzelnes Zentralatom verknüpft sind, auf zwei $\mathrm{Me}_{2} \mathrm{Si}$ - und $\mathrm{CH}_{2-}$ verbrückte Derivate beschränkt ist. ${ }^{[4,5]} \mathrm{Im}$ Gegensatz dazu sind das isoelektronische Tetranitromethan $\mathrm{C}\left(\mathrm{NO}_{2}\right)_{4}$ und Tetramethylmethantetracarboxylat $\mathrm{C}(\mathrm{COOMe})_{4}$ seit langem bekannt. ${ }^{[6]}$
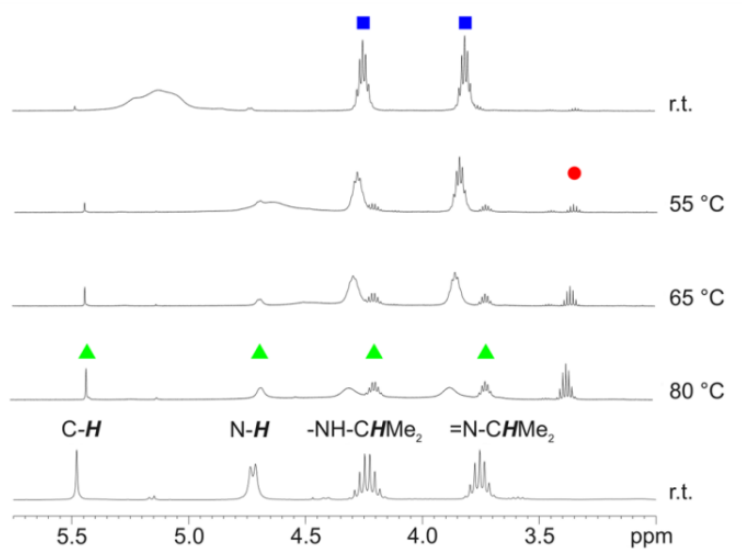

Abbildung 1. Temperaturabhängiger ${ }^{1} \mathrm{H}$ NMR-Spektrenverlauf der Reaktion von $1 \mathrm{a}$ mit Wasser in $\mathrm{C}_{6} \mathrm{D}_{6}\left(\mathbf{1 a} \mathbf{a}, \mathbf{2 a} \Delta, \mathrm{C}(\mathrm{Ni}-\mathrm{Pr})_{2} \bullet\right)$.

Wir synthetisierten kürzlich verschiedene Tetraamidinatmethankomplexe $\left\{\mathrm{C}\left[\mathrm{C}(\mathrm{NR})_{2} \mathrm{ZnMe}\right]_{4}\right\}(\mathrm{R}=i$-Pr 1a; Ph 1b, Et 1c, Cy 1d) durch Reaktion von $\mathrm{ZnMe}_{2}$ mit Carbodiimiden bei erhöhter Temperatur und untersuchten ihre Reaktivität. ${ }^{[7,8]}$ Aufgrund unseres Interesses an den entsprechenden neutralen tetradentaten Methantetra-

[*] B. Gutschank, Prof. S. Schulz*, M. Marcinkowski, Prof. G. Jansen, Dipl. Ing. H. Bandmann, D. Bläser, Dr. C. Wölper Fakultät für Chemie, Universität Duisburg-Essen Universitätsstr. 5-7, S07 S03 C30, D-45117 Essen Fax: (+) 201-183 3830

E-mail: stephan.schulz@uni-due.de

$[* *] \quad$ S. Schulz dankt der Universität Duisburg-Essen für finanzielle Unterstützung.

Weiterführende Information zu diesem Artikel sind erhältlich im WWW unter http://www.angewandte.org oder vom Autor. amidinen untersuchten wir die kinetisch-kontrollierte Hydrolyse dieser Zinkamidinatkomplexe. Temperaturabhängige in situ ${ }^{1} \mathrm{H}-$ NMR-Studien (Abb. 1) der Reaktion von 1a mit Wasser in $\mathrm{C}_{6} \mathrm{D}_{6}$ zeigten einen unerwarteten Reaktionsverlauf. 1a ist bei Raumtemperatur nahezu hydrolysestabil, während ab $55^{\circ} \mathrm{C}(i \text {-PrN })_{2} \mathrm{C}$ und das Methantrisamidin 2a gebildet werden. Obwohl das erwartete Tetraamidin $\mathrm{C}[\mathrm{C}(\mathrm{NR}) \mathrm{N}(\mathrm{R}) \mathrm{H}]_{4}$ nicht detektiert wird, kann dessen Bildung als Reaktionsintermediat nicht vollkommen ausgeschlossen werden. Vergleichbare Reaktionen wurden für die isoelektronischen $\mathrm{C}\left(\mathrm{NO}_{2}\right)_{4}$ und $\mathrm{C}(\mathrm{COOMe})_{4}$, die unter basischen Bedingungen $\mathrm{zu}$ $\mathrm{Ag}^{+}\left[\mathrm{C}\left(\mathrm{NO}_{2}\right)_{3}\right]^{-}$und $\mathrm{HC}(\mathrm{COOMe})_{3}$ abgebaut werden, gefunden. ${ }^{\left[{ }^{[9]}\right.} \mathrm{Im}$ Gegensatz dazu wurden analoge Carbodiimideliminierungsreaktionen lediglich bei der thermischen Zersetzung von Metallamidinatkomplexen $(\mathrm{Cu}, \mathrm{Al})$ bei Temperaturen oberhalb von $200{ }^{\circ} \mathrm{C}$ beobachtet. ${ }^{[10]}$ Die niedrigeren Reaktionstemperaturen bei der Zersetzung von 1a legen jedoch einen alternativen intramolekularen Zerfallsmechanismus nahe (SI).

2a wurde durch Sublimation bei $80{ }^{\circ} \mathrm{C}$ gereinigt. Das ${ }^{1} \mathrm{H}-\mathrm{NMR}$ Spektrum zeigt ein Singulett (5.37 ppm, Toluol-d8) der zentralen CH-Gruppe, während die NH-Resonanz (4.63 ppm) als Dublett aufgrund der ${ }^{3} \mathrm{~J}_{\mathrm{HH}}-\mathrm{Kopplung}$ zur $\mathrm{CH}_{i \text {-Pr }}$ Gruppe erscheint. Dynamische ${ }^{1} \mathrm{H}-\mathrm{NMR}$ und DEPT Experimente zwischen -40 und $+100{ }^{\circ} \mathrm{C}$ zeigten keine $\mathrm{CH}-\mathrm{NH}$ Tautomerisierung der zentralen $\mathrm{C}-\mathrm{H}$ Einheit. Im Gegensatz dazu bilden sich bei der Hydrolyse von $\mathbf{1 b}$ mit $\left[\mathrm{C}(\mathrm{NPh}) \mathrm{N}(\mathrm{Ph})_{\mathrm{H}}\right]_{2}\left[\mathrm{C}(\mathrm{HNPh})_{2}\right] \mathbf{2 b}$ und $\mathrm{HC}[\mathrm{C}(\mathrm{NHPh}) \mathrm{NPh}]_{3} \mathbf{2 c}$ beide tautomeren Formen. $\mathbf{2 b}$ und $\mathbf{2 c}$ repräsentieren die ersten strukturell charakterisierten $\mathrm{CH}-\mathrm{NH}$ Tautomere eines azyklischen Amidins. Obwohl die N,N'-Tautomerisation von Amidinen in der Vergangenheit intensiv untersucht wurde, ${ }^{[11]}$ wurde die Existenz eines $\mathrm{CH}-\mathrm{NH}$ Tautomerengleichgewichts lediglich indirekt bewiesen durch das Auftreten einer kurzlebigen En-1,1-Diamin Spezies, welche jedoch nicht isoliert werden konnte. ${ }^{[12]}$ Dagegen wurde ein zyklisches En1,1-diamin NMR-spektroskopisch charakterisiert. ${ }^{[13]}$ Das postulierte $\mathrm{N}-\mathrm{H}$-Tautomer von Acetamidin spielt eine zentrale Rolle in der Diels-Alder-Reaktion von Tetrazinderivaten zu Aminopyridazinen, ${ }^{[14]}$ während ein zyklische Keten-N,N-acetal als Schlüsselkomponente bei der Synthese von Tetraazafulvalenen durch oxidative Kupplung angesehen wird. ${ }^{[15]}$

Farblose Einkristalle wurden aus einer Lösung in Cyclohexan $/ \mathrm{CH}_{2} \mathrm{Cl}_{2}$ (2b) bzw. durch Lagerung einer $\mathrm{CH}_{3} \mathrm{CN}$-Lösung bei $10{ }^{\circ} \mathrm{C}(\mathbf{2 c})$ erhalten. ${ }^{[16]}$ Das sp ${ }^{2}$-hybridisierte C1-Atom in $\mathbf{2 b}$ ist von drei weiteren C-Atomen in einer trigonal-planaren Anordnung umgeben (r.m.s. Abweichung der vier C-Atome von der besten Ebene beträgt $0.0044 \AA$ ). Die C1-C2 und C1-C3 Bindungen sind kürzer als eine typische C-C-Einfachbindung (1.54 $\AA$ ), während der C1-C4 Abstand gegenüber einer C-C-Doppelbindung (1.34 $\AA$ ) aufgeweitet ist, was auf eine Delokalisation der $\pi$-Elektronen hinweist. Analoge Beobachtungen werden für beide $\mathrm{C} 4-\mathrm{N}$ Bindungen gemacht, die jeweils kürzer als eine typische $\mathrm{C}\left(\mathrm{sp}^{2}\right)-\mathrm{N}\left(\mathrm{sp}^{3}\right)$ Einfachbindung sind $(1.43 \AA)$. Im Gegensatz dazu liegen je eine C-N Bindung an $\mathrm{C} 2$ und C3 im Bereich einer C-N Doppelbindung (1.29 $\AA$ ), während die beiden anderen gut mit dem Mittelwert einer C-N Einfachbindung für $\mathrm{R}(\mathrm{H}) \mathrm{N}-\mathrm{CR}=\mathrm{NR}$ Einheiten übereinstimmen (CSD-Suche; 1.372(28) §). Die Strukturparameter des Moleküls passen sehr gut 
zu der H-Position, die in der Differenz-Fourier-Synthese gefunden wurde. Die Konformation des Moleküls wird durch zwei intramolekulare Wasserstoffbrückenbindungen unterstützt.

Das zentrale Kohlenstoffatom (C1) in $\mathbf{2 c}$ bindet an drei Amidineinheiten und ein weiteres H-Atom, welches in der DifferenzFourier-Synthese eindeutig gefunden wurde und dessen Position im Einklang mit den übrigen Strukturparametern steht. Die C-CBindungslängen sind im Bereich typischer $\mathrm{C}-\mathrm{C}$-Einfachbindungen und die $\mathrm{C}-\mathrm{C} 1-\mathrm{C}$ Bindungswinkel betragen ca. $113^{\circ}$. $\mathrm{C} 1$ ragt leicht aus der $\mathrm{C} 2 / \mathrm{C} 3 / \mathrm{C} 4$-Ebene heraus $(0.4180(12) \AA)$, was auf eine $\mathrm{sp}^{3}$ Hybridisierung hinweist, wobei die leicht vergrößerten C-C1-C Bindungswinkel auf den größeren sterischen Anspruch der Amidingruppen zurückzuführen sind. Die C-N Bindungslängen liegen im typischen Bereich für Einfach- und Doppelbindungen.

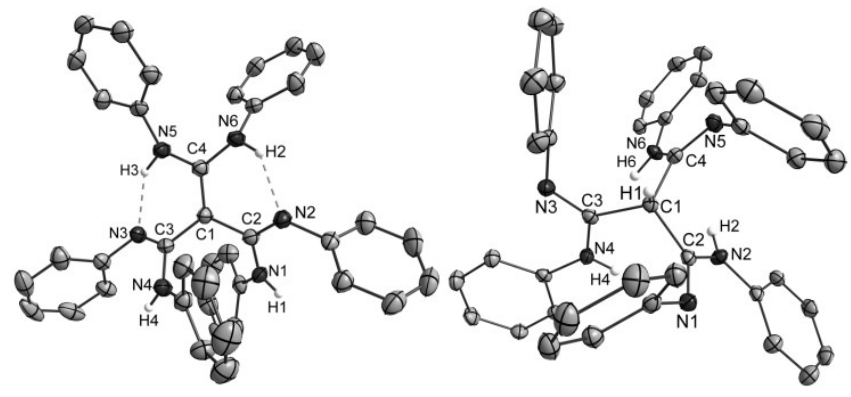

Abbildung 2. Molekülstruktur von 2b (links) und 2c (rechts); Thermalellipsoide mit 50\% Aufenthaltswahrscheinlichkeit, Wasserstoffatome mit frei gewähltem Radius, Phenyl-H-Atome sind zur besseren Übersicht nicht gezeigt. Bindungslängen $[\AA]]$ und -winkel $\left[{ }^{\circ}\right]$ : $2 \mathbf{b}$ : C(1)$\mathrm{C}(2)$ 1.486(2), C(1)-C(3) 1.476(2), C(1)-C(4) 1.391(2), N(1)-C(2) 1.374(2), N(2)-C(2) 1.294(2), N(5)-C(4) 1.372(2), N(6)-C(4) 1.373(2), $\mathrm{C}(4)-\mathrm{C}(1)-\mathrm{C}(3)$ 121.38(13), C(4)-C(1)-C(2) 120.64(14), C(3)-C(1)-C(2) 117.97(14), N(2)-C(2)-N(1) 122.51(14), N(5)-C(4)-N(6) 119.16(14);

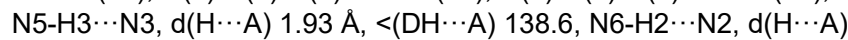
$1.97 \AA,<(\mathrm{DH} \cdots \mathrm{A})$ 137.0; 2c: C(1)-C(2) 1.5356(14), C(1)-C(3) 1.5345(14), C(1)-C(4) 1.5341(15), N(1)-C(2) 1.2819(14), N(2)-C(2) 1.3644(14), C(4)-C(1)-C(3) 113.04(8), C(4)-C(1)-C(2) 112.37(9), C(3)$\mathrm{C}(1)-\mathrm{C}(2) 113.21(9), \mathrm{N}(1)-\mathrm{C}(2)-\mathrm{N}(2) 121.88(10)$.

Zur Untersuchung einer möglichen Lösungsmittelabhängigkeit der Gleichgewichtslage von $\mathbf{2 b}$ und $\mathbf{2 c}$ wurden temperaturabhängige NMR-Experimente in $\mathrm{C}_{6} \mathrm{D}_{6}$ und $\mathrm{CD}_{3} \mathrm{CN}$ durchgeführt. In unpolaren $\mathrm{C}_{6} \mathrm{D}_{6}$ liegt vorzugsweise $\mathbf{2 b}(>85 \%$ ) über den kompletten Temperaturbereich $\left(25-75{ }^{\circ} \mathrm{C}\right)$ vor. Die ${ }^{13} \mathrm{C}$ - und DEPT90-Spektren zeigen lediglich vier verbreiterte Phenylresonanzen aufgrund des schnellen intramolekularen Protonenaustauschs zwischen der Amino- und Iminogruppe. Die C-C-Doppelbindung ist demzufolge über die gesamte planare $\mathrm{C}_{4}$-Einheit delokalisiert, was die ausgesprochene Stabilität von $\mathbf{2 b}$ erklärt. Das ${ }^{13} \mathrm{C}$ NMR Spektrum von $\mathbf{2 b}$ zeigt charakteristische Resonanzen des Endiamidin-C-Atoms (84.96 ppm), der Amidin-C-Atome (153.74 ppm) und des Endiamin-C-Atoms (143.53 ppm). Dagegen zeigen die NMR-Spektren von $\mathbf{2 b}$ in polaren $\mathrm{CD}_{3} \mathrm{CN}$ beim Erwärmen von $25 \mathrm{C}$ auf $75{ }^{\circ} \mathrm{C}$ eine relative $\mathrm{Zu}$ nahme von 2c. Da aufgrund der schlechten Löslichkeit von $2 \mathbf{c}$ aber hohe Temperaturen erforderlich sind, weist dies eher auf einen Lösungsmitteleffekt (z.B. Polarität) als auf eine Temperaturabhängigkeit hin. Die relative Konzentration beider Tautomere wird demnach teilweise durch die Polarität des Lösungsmittels beeinflusst. ${ }^{[17]}$

Die relative Stabilität der N-H (2b) und C-H (2c) Tautomere wurden mittels dispersionskorrigierter Dichtefunktionaltheorie $(\mathrm{DFT}+\mathrm{D} 3)$ untersucht. ${ }^{[18]}$ Nach Konvergenz der Geometrieoptimierungen wies $\mathbf{2} \mathbf{b} \mathrm{C}_{2}$-Symmetrie auf, während $\mathbf{2} \mathbf{c} \mathrm{C}_{3}$-Symmetrie zeigte.
In 2c wird die Methingruppe von Phenylgruppen mit $\mathrm{H}_{\text {ortho- }} \mathrm{C}_{\text {meta- }}$ Abständen von $2.87 \AA$ umgeben (XRD 2.98, 3.02, $3.13 \AA$ ), was den stabilisierenden Einfluss der drei $\mathrm{CH}-\pi$-Kontakte belegt. Der berechnete C1-C2-Abstand (1.545 $\AA$ ) und der C2-C1-C3-Winkel $\left(113.2^{\circ}\right)$ stimmen sehr gut mit der Kristallstruktur überein. Die verbleibenden drei Phenylgruppen zeigen keine $\mathrm{CH}$ - $\pi$-oder $\pi-\pi$ Stabilisierungen, aber je eines ihrer $\mathrm{H}_{\text {ortho-Atome ist Teil eines nahe- }}$ zu planaren $\mathrm{H}-\mathrm{C}-\mathrm{C}-\mathrm{N}-\mathrm{C}-\mathrm{N}-\mathrm{Sechsrings}$ und lediglich $2.28 \AA$ vom benachbarten N-Atom entfernt. Drei $\mathrm{NH} \cdots \mathrm{N}-$ Kontakte $(2.55 \AA)$ zwischen den $\mathrm{C}(\mathrm{NHPh})(\mathrm{NPh})-$ Gruppen führen $\mathrm{zu}$ einer weiteren Stabilisierung dieser Struktur. 2b zeigt zwei CH- $\pi$-Kontakte mit $\mathrm{H}_{\text {ortho-}} \mathrm{C}_{\text {para- }}$ Abständen von $2.87 \AA$ (XRD 2.87, $2.90 \AA$ ), einen $\pi$ - $\pi$ Kontakt in der typischen parallelverschobenen Anordnung zweier Phenylringe an benachbarten $\mathrm{C}(\mathrm{NHPh})(\mathrm{NPh})$-Resten und zwei 1.80 $\AA$ kurze $\mathrm{NH} \cdots \mathrm{N}$-Bindungen (XRD 1.93, $1.97 \AA$ ), die $\mathbf{2 b}$ gegenüber 2c um $10.6 \mathrm{~kJ} / \mathrm{mol}$ stabilisieren. Der berechnete C1-C2-Abstand $(1.475 \AA)$ stimmt sehr gut mit den experimentellen Werten (C1-C2 1.486(2)), C1-C3 1.476(2) Å) überein, während der C1-C4-Abstand von $1.420 \AA$ auf einen etwas höheren Einfachbindungsanteil der berechneten Struktur hinweist (XRD 1.391(2) Å). Die DFT+D3 Energiedifferenz stimmt gut mit dem in ab initio-Rechnungen mittels Møller-Plesset-Störungstheorie zweiter Ordnung (MP2) für die DFT+D3-Geometrien gefundenem Wert von $12.3 \mathrm{~kJ} / \mathrm{mol}$ überein. ${ }^{[19]}$ Unter Berücksichtigung des auf DFT+D3-Niveau erhaltenen größeren Dipolmoments von 2c (2.42 Debye) im Vergleich zu 2b (0.97 Debye) ist diese Energiedifferenz klein genug um zu erklären, warum $\mathbf{2 b}$ vorzugsweise, aber nicht ausschließlich, in unpolaren Lösungsmitteln beobachtet wird, während $2 \mathbf{c}$ in polaren Solventien dominiert (vide supra).

Ersatz der Phenylgruppen durch H-Atome gefolgt von Geometrieoptimierung und Schwingungsfrequenzberechnung auf DFT+D3Niveau lieferte ein $\mathrm{C}_{2}$-symmetrisches $\mathrm{N}-\mathrm{H}-$ und ein $\mathrm{C}_{3-}$ symmetrisches $\mathrm{C}-\mathrm{H}-\mathrm{Tautomer}$ als echte Minima auf der Potentialenergiefläche. ${ }^{[20]}$ Das N-H-Tautomer ist mit $23.8 \mathrm{~kJ} / \mathrm{mol}$ energetisch bevorzugt, in guter Übereinstimmung mit der MP2Energiedifferenz von $22.1 \mathrm{~kJ} / \mathrm{mol}$ für die DFT+D3-Geometrien, welche sich bei Nachoptimierung der Molekülstrukturen auf dem MP2-Niveau nur leicht auf $23.1 \mathrm{~kJ} / \mathrm{mol}$ verändert. Der $\pi$ - $\pi$-Kontakt zwischen benachbarten $\mathrm{C}(\mathrm{NHPh})(\mathrm{NPh})-\mathrm{Gruppen}$, der in $\mathbf{2 b}$ beobachtet wurde, wird durch zwei NH $\cdots \mathrm{N}-$ Kontakte mit einem $\mathrm{N} \cdots \mathrm{H}-$ Abstand von $2.50 \AA$ ersetzt. Das Fehlen von $\mathrm{CH}-\pi$-Kontakten erklärt die größere Energiedifferenz zwischen beiden Tautomeren verglichen mit 2b/2c. Nach Aufhebung der Beschränkung auf $\mathrm{C}_{3^{-}}$ Symmetrie wurde für das C-H-Tautomer eine zweite, energetisch tieferliegende $\mathrm{C}_{1}$-symmetrische Struktur gefunden (siehe SI). Auf DFT+D3-Niveau liegt diese Struktur um $20.9 \mathrm{~kJ} / \mathrm{mol}$ über dem NH-Tautomer, während sie auf dem MP2-Niveau lediglich um 16.1 $\mathrm{kJ} / \mathrm{mol}$ höher liegt $(17.1 \mathrm{~kJ} / \mathrm{mol}$ nach MP2-Nachoptimierung beider Strukturen).

Zur Bestimmung des Energieunterschieds zwischen dem (unbeobachteten) $\mathrm{N}-\mathrm{H}-$ und dem C-H-Tautomer (2a) des $i$-Prsubstituierten Trisamidins wurde ein Konformerenscan mit einem Molecular-Mechanics-Kraftfeld für beide Tautomere durchgeführt. Die 12 energetisch günstigsten N-H-Tautomere und die 15 niedrigsten C-H-Tautomere wurden dann auf DFT+D3-Niveau mit einem Basissatz von split-valence-Qualität nachoptimiert. Jeweils zwei der energetisch tiefliegensten Tautomerenstrukturen wurden anschließend auf DFT+D3-Niveau mit einem triple-zeta Basissatz reoptimiert. Ein $\mathrm{C}_{1}$-symmetrisches Konformer von 2a liegt um 1.9 $\mathrm{kJ} /$ mol niedriger als alle $\mathrm{N}-\mathrm{H}$-Tautomerenkonformere, deren tiefstliegendstes $\mathrm{C}_{2}$-Symmetrie aufweist. Das nächste Konformer von 2a $\left(\mathrm{C}_{1}\right.$-Symmetrie) wurde bei $2.0 \mathrm{~kJ} / \mathrm{mol}$ gefunden, während die Ener- 
gie des nächsten N-H-Tautomerenkonformers $\left(\mathrm{C}_{1}\right)$ um $5.6 \mathrm{~kJ} / \mathrm{mol}$ über dem tiefsten Konformer von 2a liegt. Die Energiedifferenz zwischen den niedrigsten Konformeren beider Tautomere wächst auf MP2-Niveau auf $3.3 \mathrm{~kJ} / \mathrm{mol}$ an (ohne Nachoptimierung der DFT+D3-Strukturen), was aber zu klein ist um die experimentell beobachtete ausschließliche Bildung von 2a zu erklären. Allerdings beträgt das Dipolmoment von 2a 2.42 Debye (DFT+D3), während das der beiden N-H-Tautomerenkonformere mit 1.02 bzw. 1.07 Debye deutlich kleiner ist. Die bevorzugten Bildung des $\mathrm{C}-\mathrm{H}-$ Tautomers 2a resultiert daher vermutlich aus Wechselwirkungen mit benachbarten Dipolen oder einer polarisierbaren Umgebung.

Die Iminfunktionen der Methantrisamidine sind gute Protonenakzeptoren, was die Reaktion von $\mathbf{2 b}$ mit zwei Äquivalenten Essigsäure $\mathrm{zu}\left[\mathrm{C}\left(\mathrm{C}(\mathrm{HNPh})_{2}\right)_{3}\right]^{2+}\left\{\left[\mathrm{CH}_{3} \mathrm{COO}^{-}\right\}_{2} 3\right.$ belegt (SI). Kristalle von 3, welches in der triklinen Raumgruppe $P$ 1 kristallisiert, wurden aus einer Lösung in $\mathrm{Et}_{2} \mathrm{O}$ bei $-30{ }^{\circ} \mathrm{C}$ erhalten. Der auffälligste Strukturunterschied zwischen $\mathbf{2 b}$ und dem Methantrisamidiniumdikation 3 liegt in den nahezu gleichlangen C-C-Bindungslängen innerhalb der trigonal-planaren $\mathrm{C}_{4}$-Einheit (C1-C2 1.417(2) $\AA$, C1-

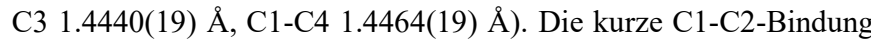
kann durch die Orientierung der N-C-N-Einheit relativ zur zentralen $\mathrm{C}_{4}$-Einheit erklärt werden. Die N1/C2/N2-Ebene liegt nahezu koplanar zur $\mathrm{C} 1 / \mathrm{C} 2 / \mathrm{C} 3 / \mathrm{C} 4$-Ebene $\left(26.51(15)^{\circ}\right)$, was eine effektivere $\pi$-Elektronendelokalisation im Vergleich $\mathrm{zu}$ den beiden anderen Amidinatgruppen $\left(40.27(13)^{\circ}, 43.38(10)^{\circ}\right)$ erlaubt und die leicht verlängerten $\mathrm{C} 2-\mathrm{N}-\mathrm{Bindungen}$ sowie die leicht verkürzte $\mathrm{C} 1-\mathrm{C} 2-$ Bindung erklärt.

Die polaren N-H-Gruppen in 2a-c lassen sich zudem metallieren. So reagiert 2a mit $\mathrm{AlMe}_{3}$ und $i$-Bu2 $\mathrm{AlH}$ unter Eliminierung von Methan bzw. $\mathrm{H}_{2}$ und quantitativer Bildung von $\mathrm{HC}\left[\mathrm{C}(\mathrm{N} i \text { - } \mathrm{Pr})_{2} \mathrm{AlR}_{2}\right]_{3}$ $\left(\mathrm{R}=\mathrm{Me}\right.$ 4a, $i$-Bu 4b), deren Identität mittels ${ }^{1} \mathrm{H}-\mathrm{NMR}$ Spektroskopie $\left(\mathrm{C}_{6} \mathrm{D}_{6}\right)$ durch das Fehlen der NH-Resonanz und dem Auftreten der charakteristischen CH-Resonanz belegt wurde. Kristalle von 4a und 4b wurden aus verschiedenen Solventien erhalten, wobei ihre geringe Qualität nur Aussagen zur Konnektivität zuließ. Das zentrale C-Atom ist jeweils $\mathrm{sp}^{3}$-hybridisiert und die Amidinatgruppen binden chelatisierend an die $\mathrm{AlR}_{2}$-Einheit.

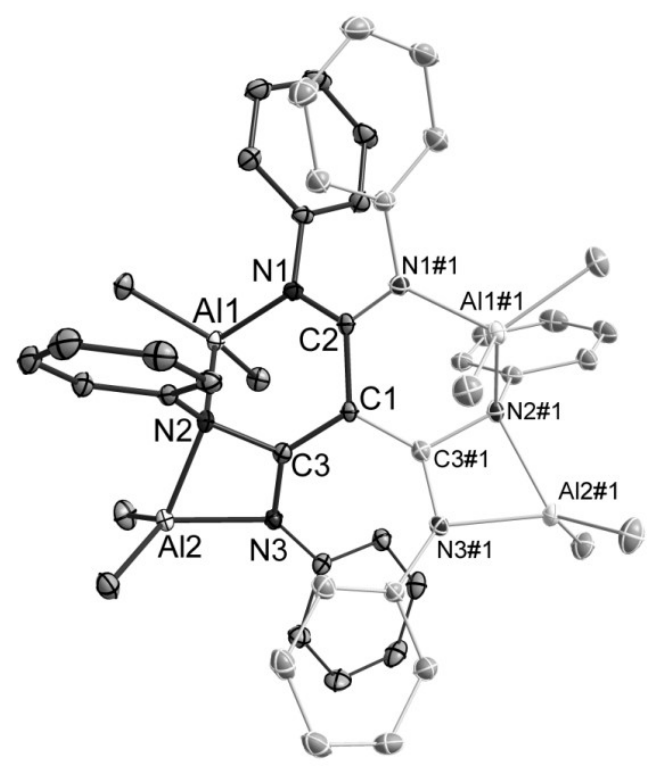

Figure 3. Molekülstruktur von 4c (Thermalellipsoide mit 50\% Aufenthaltswahrscheinlichkeit, Wasserstoffatome sind zur besseren Übersicht nicht gezeigt, die asymmetrische Einheit mit dunklen Bindungen gezeigt, $C 1$ und $C 2$ liegen auf einer Zweizähligen Achse). Bindungslängen $[\AA]]$ und -winkel $\left[^{\circ}\right], \# 1-x+1, y,-z+1 / 2$ : $C(1)-C(2) 1.506(2), C(1)-$
$\mathrm{C}(3) 1.4191(15), \mathrm{N}(2)-\mathrm{C}(3) 1.4470(15), \mathrm{N}(3)-\mathrm{C}(3)$ 1.3257(16), N(1)$\mathrm{C}(2)$ 1.3404(13), Al(1)-N(1) 1.9199(11), Al(1)-N(2) 1.9984(11), Al(2)$\mathrm{N}(3)$ 1.9268(11), Al(2)-N(2) 1.9967(11), C(3)\#1-C(1)-C(3) 120.55(16), $\mathrm{C}(3) \# 1-\mathrm{C}(1)-\mathrm{C}(2)$ 119.73(8), C(3)-C(1)-C(2) 119.73(8), N(1)-C(2)$\mathrm{N}(1) \# 1$ 125.02(16), N(1)-C(2)-C(1) 117.49(8), N(3)-C(3)-C(1) 132.19(12), N(3)-C(3)-N(2) 105.46(10), C(1)-C(3)-N(2) 122.29(11), $\mathrm{C}(2)-\mathrm{N}(1)-\mathrm{C}(4)$ 120.32(11), C(2)-N(1)-Al(1) 125.64(9), C(4)-N(1)-Al(1) 113.22(8), C(3)-N(2)-C(10) 120.94(10), C(3)-N(2)-Al(2) 89.32(7), $\mathrm{C}(10)-\mathrm{N}(2)-\mathrm{Al}(2)$ 112.40(8), C(3)-N(2)-Al(1) 101.14(7), C(10)-N(2)$\mathrm{Al}(1)$ 112.80(8), $\mathrm{Al}(2)-\mathrm{N}(2)-\mathrm{Al}(1)$ 118.29(5), C(3)-N(3)-C(16) 132.96(11), C(3)-N(3)-Al(2) 96.09(8), C(16)-N(3)-Al(2) 129.65(8), $\mathrm{N}(1)-\mathrm{Al}(1)-\mathrm{N}(2)$ 90.67(5), N(3)-Al(2)-N(2) 68.46(4).

Im Gegensatz dazu reagiert 2b mit der vierfachen Menge $\mathrm{AlMe}_{3}$ quantitativ zu $\mathrm{C}\left[\mathrm{C}(\mathrm{NPh})_{2} \mathrm{AlMe}_{2}\right]_{2}\left[\mathrm{C}\left(\mathrm{N}(\mathrm{Ph}) \mathrm{AlMe}_{2}\right)_{2}\right] \quad$ 4c. Gelbe Kristalle von $4 \mathbf{c}$ wurden aus einer Lösung in Toluol bei $-30{ }^{\circ} \mathrm{C}$ erhalten. $4 \mathbf{c}$ kristallisiert in der monoklinen Raumgruppe $C 2 / c$ mit $\mathrm{C} 1$ und $\mathrm{C} 2$ auf einer zweizähligen Drehachse. Eine Amidinatgruppe (N1-C2-N1\#1) verbrückt zwei AlMe2-Einheiten, während die beiden anderen Amidinatgruppen je eine AlMe2-Gruppe chelatisieren. Die C-Atome der Amidinatgruppen binden in einer trigonalplanaren Anordnung an das zentrale C-Atom $(\mathrm{C} 1)$, wobei es zur Bildung einer langen und (symmetriebedingt) zweier kurzer C-CBindungen kommt. Die $\pi$-Elektronen sind über die gesamte N3-C3C1-C3\#1-N3\#1 Einheit delokalisiert. Die C-N-Bindungslängen innerhalb dieser beiden Amidinatgruppen differieren aufgrund der unterschiedlichen Koordinationszahlen der N-Atome, während die identischen C-N-Bindungslängen innerhalb der N1-C2-N1\#1 Einheit auf ein delokalisiertes $\pi$-Elektronensystem hinweisen.

Die Methantrisamidine $\left\{\mathrm{HC}[\mathrm{C}(\mathrm{NR}) \mathrm{NHR}]_{3}(\mathrm{R}=i\right.$-Pr 2a, Ph 2c) und En-1,1-Diamidin-2,2-diamin $\left.\left\{\mathrm{C}[\mathrm{C}(\mathrm{NPh}) \mathrm{NHPh})_{2}\right]_{2}\left[\mathrm{C}(\mathrm{NHPh})_{2}\right]\right\}$ 2b wurden durch Hydrolyse der entsprechenden tetranuklearen Zinkkomplexe in einer bislang unbeobachteten Carbodiimideliminierungsreaktion synthetisiert. Mit den N-H- (2b) und C-HTautomeren (2c) gelang erstmalig der strukturelle Nachweis der N,C-Tautomerie in Amidinen. Die relative Gleichgewichtslage von 2b und 2c kann in Lösung durch die Polarität des Lösungsmittels beeinflusst werden. Quantenchemische Rechnungen belegen, dass das N-H-Tautomer im Falle der Ph- und H-substituierten Trisamidine jeweils energetisch gegenüber dem $\mathrm{C}-\mathrm{H}$-Tautomer begünstigt ist, während im Falle des $i$-Pr-substituierten Komplexes das $\mathrm{C}-\mathrm{H}-$ Tautomer energetisch gegenüber dem N-H-Tautomer leicht bevorzugt ist. Reaktivitätsstudien belegen, dass sich diese neuartigen Liganden einerseits an den Lewis-basischen Imin-N-Atomen protonieren lassen, andererseits die N-H-Funktionen durch Reaktionen mit metallorganischen Verbindungen metalliert werden können.

\section{Experimenteller Teil}

Experimentelle Details befinden sich im elektronischen Anhang.

Received: ((will be filled in by the editorial staff))

Published online on ((will be filled in by the editorial staff))

Keywords: Tautomerie $\cdot$ Einkristallstrukturanalyse $\cdot$ ab-initio Berechnungen $\cdot \operatorname{Imin} \cdot$ Enamin

[1] a) J. Barker, M. Kilner, Coord. Chem. Rev. 1994, 133, 219; b) F. T. Edelmann, Adv.Organomet. Chem. 2008, 57, 1535; c) M. P. Coles, Dalton. Trans. 2006, 118, 985. 
[2] a) K. A. Schug, W. Lindner, Chem. Rev. 2005, 105, 67; b) M. W. Hosseini, Coord. Chem. Rev. 2003, 240, 157.

[3] a) C. Nastruzzi, R. Gambari, Cancer Lett. 1990, 50, 93-102; b) R. R. Tidwell, L. L. Fox, J. D. Geratz, Biochim. Biophys. Acta 1976, 445, 729-738.

[4] a) J.-A. Gautier, M. Miocque, C. C. Farnoux, in The Chemistry of Amidines and Imidates, Vol. 1 (Eds: S. Patai), JOHN WILEY \& SONS, London, 1975, pp. 283-348. b) R. L. Shriner, F. W. Neumann, Chem. Rev, 1944, 35(3), 351.

[5] a) A. Kraft, R. Fröhlich, Chem. Commun. 1998, 1085; b) A. Kraft, Perkin Trans. 1999, 1, 705; c) S. K. Mandal, L. K. Thompson, M. J. Newland, E. J. Gabe, F. L. Lee, Chem. Commun. 1989, 744; d) S. S. Tandon, L. K. Thompson, J. N. Bridson, J. C. Dewan, Inorg. Chem. 1994, 33, 54; e) A. W. Cordes, R. C. Haddon, R. G. Hicks, R. T. Oakley, T. T. M. Palstra, L. F. Schneemeyer, J. V. Waszczak, J. Am. Chem. Soc. 1992, 114, 5000; f) R. T. Boeré, R. T. Oakley, R. W. Reed, J. Organomet. Chem. 1987, 331, 161; g) J. Grundy, M. P. Coles, P. B. Hitchcock, J. Organomet. Chem. 2002, 662, 178; h) J. R. Hagadorn, J. Arnold, Angew. Chem. 1998, 110, 1813; Angew. Chem. Int. Ed. 1998, 37, 1729; i) H. Kawaguchi, T. Matsuo, Chem. Commun. 2002, 958; j) J. F. Li, L. H. Weng, X. H. Wei, D. S. Liu, Dalton Trans. 2002, 1401; k) C. T. Chen, L. H. Rees, A. R. Cowley, M. L. H. Green, Dalton Trans. 2001, 1761; 1) H. A. Jenkins, D. Abeysekera, D. A. Dickie, J. A. C. Clyburne, Dalton Trans. 2002, 3919; m) S.-D. Bai, J.-P. Guo, D.-S. Liu, W.-Y. Wong, Eur. J. Inorg. Chem. 2006, 4903; n) J. R. Babcock, C. Incarvito, A. L. Rheingold, J. C. Fettinger, L. R. Sita, Organometallics 1999, 18, 5729.

[6] a) F. D. Chattaway, J. Chem. Soc., Trans., 1910, 97, 2099-2102; b) R. Scholl, W. Egerer, Liebigs Ann. Chem. 1913, 397, 301.

[7] a) M. Münch, U. Flörke, M. Bolte, S. Schulz, D. Gudat, Angew. Chem. 2008, 120, 1535; Angew. Chem. Int Ed. 2008, 47, 1512; b) S. Schmidt, S. Gondzik, S. Schulz, D. Bläser, R. Boese, Organometallics 2009, 28, 4371; c) S. Schmidt, B. Gutschank, S. Schulz, D. Bläser, R. Boese, C. Wölper, Eur. J. Inorg. Chem. 2011, 28, 4464.

[8] a) B. Gutschank, S. Schulz, U. Westphal, D. Bläser, R. Boese, Organometallics 2010, 29, 2093; b) B. Gutschank, S. Schulz, D. Bläser, R. Boese, C. Wölper, Organometallics 2010, 29, 6133.

[9] a) M. Göbel, T. M. Klapötke, P. Mayer, Z. Anorg. Allg. Chem. 2006, 632, 1043; b) R. Scholl, W. Egerer, Liebigs Ann. Chem. 1913, 397, 301 .

[10] a) T. Whitehorne, J. P. Coyle, A. Mahmood, W. H. Monillas, G. Yap, S. Barry, Eur. J. Inorg. Chem. 2011, 3240. b) A. L. Brazeau, Z. Wang, C. N. Rowley, S. T. Barry, Inorg. Chem. 2006, 45, 2276.

[11] a) C. Berndt, T. Kaempchen, Chem. Ber. 1986, 119, 1101. b) J. Oszczapowicz, E. Raczynska, J. Osek, J. Magn. Reson. Chem. 1986, 24, 9. c) E. Raczynska, Perkin Trans. II 1986, 1189. c) E. Raczynska, R. Gawinecki, Trends Org. Chem. 1998, 7, 85.

[12] a) M. Pfau, M. Chiriacescu, G. Revial, Tetrahedron Lett. 1993, 34, 327. b) K. Sung, S.-H. Wu, R.-R. Wu, S.-Y. Sun, Org. Chem. 2002 , 67, 4298. c) K. Ito, Y. Kizuka, Y. Hirano, J. Heterocycl. Chem. 2005, $42,583$.

[13] J. Goerdeler, R. Sappelt, Chem. Ber. 1967, 100, 2064.

[14] H. P. Figeys, A. Mathy, A. Dralants, Synth. Commun. 1981, 11(8), 655.

[15] a) J. Brandenburg, C. Käpplinger, R. Beckert, Synthesis 1996, 1302; b) D. Müller, R. Beckert, J. Weston, W. Günther, H. Görls, M. Friedrich, Eur. J. Org. Chem. 2001, 4551.

[16] Bruker AXS D8 Kappa Diffraktometer mit APEX2 Detektor (MoK $\alpha$ Strahlung, $\lambda=0.71073 \AA ; T=100(1) \mathrm{K})$. Die Strukturen wurden mittels Direkter Methoden gelöst (SHELXS-97, G. M. Sheldrick, Acta Crystallogr. Sect. A 1990, 46, 467) und gegen $\mathrm{F}^{2}$ verfeinert. Eine semiempirische Absorptionskorrektur wurde nach dem Multi-ScanVerfahren (Bruker AXS APEX2) durchgeführt. Alle Nichtwasserstoffatome wurden anisotrop verfeinert, Methylwasserstoffatome als starre Gruppen und die Wasserstoffatome mittels eines Reitermodells. Die Positionen der $\mathrm{NH}$ und $\mathrm{OH}$ Wasserstoffatome wurden aus Differenz-Fourier-Synthese entnommen und und mit Constraints verfeinert. (SHELXL-97, Program for Crystal Structure Refinement, G. M. Sheldrick, Universität Göttingen, 1997 und shelXle, A Qt GUI for SHELXL. Siehe auch: Sheldrick, G. M. Acta Crystallogr. Sect. A 2008, 64, 112; C. B. Hübschle, G. M. Sheldrick, B. Dittrich, J. Appl. Cryst. 2011, 44, 1281-1284) 2b: $\left[\mathrm{C}_{40} \mathrm{H}_{34} \mathrm{~N}_{6}\right], \mathrm{M}=598.73$, farbloser Kristall
(0.42 x $0.32 \times 0.26 \mathrm{~mm})$; monoklin, Raumgruppe $C c ; a=18.1738(16)$ $\AA, b=10.3046(9) \AA, c=18.983(2) \AA ; \alpha=90^{\circ} \beta=113.163(3)^{\circ} \gamma=$ $90^{\circ}, V=3268.4(6) \AA^{3} ; Z=4 ; \mu=0.073 \mathrm{~mm}^{-1} ; \rho_{\text {ber. }}=1.217 \mathrm{~g} \mathrm{~cm}^{-3}$; 37607 Reflexe $\left(2 \theta_{\max }=59^{\circ}\right), 8129$ unabhängig $\left(R_{\text {int }}=0.0333\right) ; 415$ Parameter, Flack-Parameter $x=-0.7(15)$; größtes max./min. in der finalen Differenz-Fourier-Synthese $0.249 \mathrm{e} \AA^{-3} /-0.245 \mathrm{e} \AA^{-3} ;$ max. $/ \mathrm{min}$. Transmission $0.75 / 0.68 ; R_{1}=0.0435(\mathrm{I}>2 \sigma(\mathrm{I})), w R_{2}($ all data $)=$ 0.1076 . Aufgrund der hohen Standardabweichung von $x$ konnte die absolute Struktur nicht zuverlässig bestimmt werden. $2 \mathbf{c}$ : $\left[\mathrm{C}_{40} \mathrm{H}_{34} \mathrm{~N}_{6}\right.$ * $\left.\mathrm{C}_{2} \mathrm{H}_{3} \mathrm{~N}\right], \mathrm{M}=639.79$, farbloser Kristall $(0.24 \times 0.18 \times 0.13 \mathrm{~mm})$; triklin, Raumgruppe $P-1 ; a=12.3438(7), b=13.3509(7), c=$ $13.4235(8) \AA ; \alpha=60.586(2)^{\circ}, \beta=67.453(3)^{\circ} \gamma=66.088(3)^{\circ}, V=$ $1713.77(17) \AA^{3} ; Z=2 ; \mu=0.075 \mathrm{~mm}^{-1} ; \rho_{\text {ber. }}=1.240 \mathrm{~g} \mathrm{~cm}^{-3} ; 28355$ Reflexe $\left(2 \theta_{\max }=61^{\circ}\right), 10184$ unabhängig $\left(R_{\text {int }}=0.0234\right)$; 442 Parameter; größtes max./min. in der finalen Differenz-Fourier-Synthese 0.394 $\mathrm{e} \AA^{-3} /-0.222 \mathrm{e} \AA^{-3} ;$ max./min. Transmission $0.75 / 0.67 ; R_{1}=0.0460$ (I > $2 \sigma(\mathrm{I})), w R_{2}$ (all data) $=0.1192 .3:\left[\mathrm{C}_{40} \mathrm{H}_{36} \mathrm{~N}_{6}, 2\left(\mathrm{C}_{2} \mathrm{H}_{3} \mathrm{O}_{2}\right), 2\left(\mathrm{H}_{2} \mathrm{O}\right)\right], \mathrm{M}$ $=754.87$, schwach gelber Kristall $(0.18 \times 0.15 \times 0.12 \mathrm{~mm})$; triklin, Raumgruppe $P-1 ; a=10.5079(11) \AA, b=13.5325(15) \AA, c=$ $16.2636(19) \AA ; \alpha=110.849(5)^{\circ}, \beta=92.152(5)^{\circ}, \gamma=111.876(5)^{\circ}, V=$ 1966.6(4) $\AA^{3} ; Z=2 ; \mu=0.086 \mathrm{~mm}^{-1} ; \rho_{\text {ber. }}=1.275 \mathrm{~g} \mathrm{~cm}^{-3} ; 33622 \mathrm{Re}-$ flexe $\left(2 \theta_{\max }=50^{\circ}\right), 6990$ unabhängig $\left(R_{\text {int }}=0.0405\right)$; 505 Parameter; größtes max./min. in der finalen Differenz-Fourier-Synthese 0.229 $\mathrm{e} \AA^{-3} /-0.263 \mathrm{e} \AA^{-3} ;$ max. $/$ min. Transmission $0.75 / 0.69 ; R_{1}=0.0355$ (I > $2 \sigma(\mathrm{I})), w R_{2}$ (all data) $=0.0883$. Die Wasserstoffatome an C72 und C82 wurden als idealisiert über zwei Positionen fehlgeordnete Methylgruppen verfeinert. 4c: $\left[\mathrm{C}_{48} \mathrm{H}_{54} \mathrm{Al}_{4} \mathrm{~N}_{6}\right], \mathrm{M}=822.89$, gelber Kristall (0.25 x $0.10 \times 0.08 \mathrm{~mm})$; monoklin, Raumgruppe $C 2 / c ; a=$ 17.4755(9) $\AA, b=15.5339(8) \AA, c=18.5007(11) \AA ; \alpha=\gamma=90^{\circ}, \beta=$ $116.831(2)^{\circ}, V=4481.6(4) \AA^{3} ; Z=4 ; \mu=1.145 \mathrm{~mm}^{-1} ; \rho_{\text {ber. }}=1.220 \mathrm{~g}$ $\mathrm{cm}^{-3} ; 23163$ Reflexe $\left(2 \theta_{\max }=61^{\circ}\right), 6861$ unabhängig $\left(R_{\text {int }}=0.0334\right)$; 263 Parameter; größtes max./min. in der finalen Differenz-FourierSynthese $0.499 \mathrm{e}^{-3} /-0.235 \mathrm{e}^{-3}$; max./min. Transmission $0.75 / 0.66$; $R_{1}=0.0398(\mathrm{I}>2 \sigma(\mathrm{I})), w R_{2}$ (all data) $=0.1087$. Die kristallographischen Daten (ohne Strukturfaktoren) der in dieser Veröffentlichung beschrieben Strukturen wurden als "supplementary publication no. CCDC-868839 (2b), CCDC-868842 (2c), CCDC-868841 (3) und CCDC-868840 (4c)" beim Cambridge Crystallographic Data Centre hinterlegt. Kopien der Daten können kostenlos bei folgender Adresse in Großbritannien angefordert werden: CCDC, 12 Union Road, Cambridge, CB21EZ (Fax: (+44)1223/336033; E-mail: deposit@ccdc.cam-ak.uk).

[17] Detaillierte Information befinden sich im elektronischen Anhang.

[18] Die Molekülgeometrien von 2b und 2c wurden ausgehend von den Einkristallstrukturdaten auf DFT Niveau unter unter Einbeziehung einer Dispersionsenergiekorrektur der dritten Generation (DFT+D3; S. Grimme, J. Antony, S. Ehrlich, H. Krieg, J. Chem. Phys. 2010, 132, 154104/1-18.) mit verschärften Konvergenzschwellen und einem verbesserten DFT-Integrationsgitter optimiert. Verwendet wurden das BLYP Austausch-Korrelationsunktional (a) P. A. M. Dirac, Proc. Roy. Soc. A 1929, 123, 714; b) J. C. Slater, Phys. Rev. 1951, 81, 385; c) A. D. Becke, Phys. Rev. A 1988, 38, 3098. d) C. Lee, W. Yang, R. G. Parr, Phys. Rev. B 1988, 37, 785.) sowie ein Basissatz von triple-zetavalence-Qualität (def2-TZVP; F. Weigend, R. Ahlrichs, Phys. Chem. Chem. Phys. 2005, 7, 3297.) mit dem zugehörigen Hilfsbasissatz (F. Weigend, Phys. Chem. Chem. Phys. 2006, 8, 1057.) in der resolutionof-the-identity-Näherung (RI) wie implementiert im Turbomole V6.3 (TURBOMOLE V6.3 2009, Universität Karlsruhe und Forschungszentrum Karlsruhe GmbH, 1989-2007, TURBOMOLE GmbH, seit 2007; erhältlich unter http://www.turbomole.com; a) R. Ahlrichs, M. Bär, M. Häser, H. Horn, C. Kölmel, Chem. Phys. Lett. 1989, 162, 165; b) O. Treutler, R. Ahlrichs, J. Chem. Phys. 1995, 102, 346; c) M. von Arnim, R. Ahlrichs, J. Chem. Phys. 1999, 111, 9183.).

[19] MP2-Berechnungen mit Korrelation der Valenzelektronen wurden in der RI-Näherung mit einem vergrößerten Orbitalbasissatz (def2TZVPP) und dem zugehörigen Hilfsbasissatz durchgeführt (F. Weigend, M. Häser, H. Patzelt, R. Ahlrichs, Chem. Phys. Letters 1998, 294, 143).

[20] P. Deglmann, K. May, F. Furche, R. Ahlrichs, Chem. Phys. Lett. 2004, $384,103$. 


\section{Methantrisamidine}

Benjamin Gutschank, Stephan Schulz, Michael Marcinkowski, Georg Jansen,

Heinz Bandmann, Dieter Bläser,

Christoph Wölper Page -

Page

Synthese, Struktur, Tautomerie und Reaktivität von Methantrisamidinen

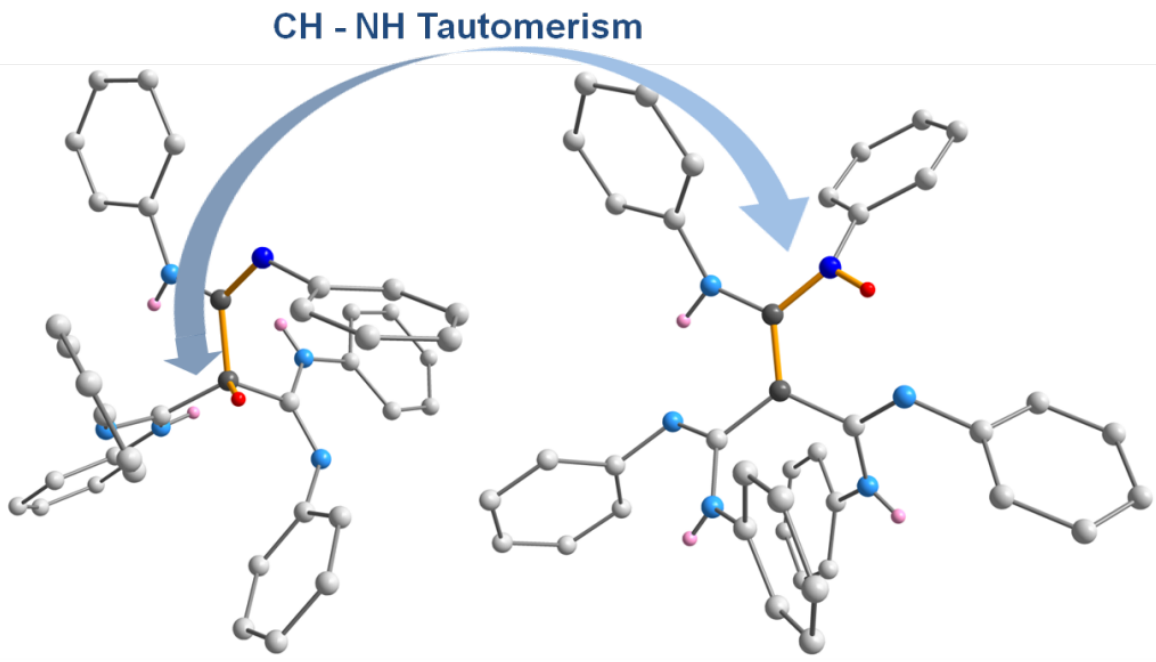

Beide tautomeren Formen eines Methantrisamidins wurden erstmalig strukturell charakterisiert und ihre vielfältige Reaktivität als Protonenakzeptoren und als multianionische Liganden demonstriert. 


\section{DuEPublico}

Duisburg-Essen Publications online
DUUESBAT R G

offen im Denken Ub \begin{tabular}{l|l} 
universitäts \\
bibliothek
\end{tabular}

This text is made available via DuEPublico, the institutional repository of the University of Duisburg-Essen. This version may eventually differ from another version distributed by a commercial publisher.

DOI: $\quad 10.1002 /$ ange.201205030

URN: urn:nbn:de:hbz:464-20201112-094323-0

This is the peer reviewed version of the following article: Angew. Chem. 2012, 24, 11051-11055 which has been published in final form at:

https://doi.org/10.1002/ange.201205030

All rights reserved. 